\title{
Pairing in Many-Fermion Systems: An Exact Renormalisation Group Treatment
}

\author{
Michael C. Birse ${ }^{1}$, Boris Krippa ${ }^{1,2}$, Judith A. McGovern ${ }^{1}$, and Niels R. Walet ${ }^{2}$ \\ ${ }^{1}$ Theoretical Physics Group, Department of Physics and Astronomy, \\ University of Manchester, M13 9PL, UK and \\ ${ }^{2}$ Department of Physics, UMIST, P.O. Box 88, Manchester, M60 1QD, UK
}

(Dated: November 1, 2018)

\begin{abstract}
We study the application of the exact renormalisation group to a many-fermion system with a short-range attractive force. We introduce a boson field to describe pairing effects, and take a simple ansatz for the effective action. We derive a set of approximate flow equations for the effective coupling including boson and fermionic fluctuations. The initial conditions are obtained by renormalising the interaction to fit the scattering length in vacuum. At some critical value of the running scale, the numerical solutions show a phase transition to a gapped phase. Standard results are recovered if we omit the boson loops. When boson fluctuations are included, we find that their contributions are significant only in the small-gap regime.
\end{abstract}

Attractive forces between fermions, even if they are too weak to produce two-body bound states, play a crucial role in many areas of many-body physics. In a system of fermions, any attraction, no matter how weak, can cause such particles to form correlated "Cooper pairs" at zero temperature. Examples range from superfluidity in liquid helium-3 to colour superconductivity in dense quark matter. The ground state of the system becomes qualitatively different through this pairing, as can be seen from the occurrence of a phase transition at some critical temperature. The order parameter for this is the energy gap which appears in the fermion spectrum. Within such systems we can identify two extreme limits, depending on the interaction strength. The weak-coupling regime, with no two-body bound states, manifests itself in Bardeen-Cooper-Schrieffer (BCS) superconductivity, while the strong-coupling regime, with a deeply bound state which approximates an elementary boson, corresponds to Bose-Einstein Condensation (BEC).

In this letter we study pairing within a framework inspired by modern effective field 
theory (EFT). An EFT description of phenomena is intended to be generic, independent of details of the underlying theory, and depending only on the degrees of freedom and interactions relevant at the energy scale being considered [1]. An interesting, but rather adhoc approach to combining pairing with EFT ideas is the work of Papenbrock and Bertsch 2]. A more detailed analysis of the same problem, but cast in a slightly different language, can be found in the condensed matter literature [3], and has a long history [4] (see also [5]). There is also some closely related work by Weinberg [6], concentrating on the effective potential in a field-theoretic framework.

Papenbrock and Bertsch 2] use a standard BCS approach with a contact interaction, which they relate to scattering observables by a subtractive renormalisation scheme. Their results are limited to positive chemical potential but many of their basic equations are identical to those of Marani et al. [3]. In the latter work the chemical potential $\mu$ is not tied to the Fermi momentum $p_{F}$, making it possible to study the crossover to BEC, which occurs for negative values of $\mu$. In order to find a more complete way to include many-body physics beyond the mean field, within an EFT-inspired approach, we have used a method that draws together ideas from Ref. [6] and Refs. 2, 3]. The approach is based on the use of the Exact Renormalisation Group (ERG) [7, 8, 9] .

The goal of the ERG approach is to construct the Legendre transform of the effective action: $\Gamma\left[\phi_{c}\right]=W[J]-J \cdot \phi_{c}$, where $W$ is the usual partition function in the presence of an external source $J[10]$. The action functional $\Gamma$ generates the 1PI Green's functions for small fluctuations around the ground state, and it reduces to the effective potential for homogeneous systems. Instead of trying to evaluate it directly from $W[J]$, one can use an artificial renormalisation group flow, created by introducing an artificial gap in the energy spectrum for the fields. This depends on a momentum scale $k$ since we can define the effective action by integrating over components of the fields with $q \gtrsim k$. The RG trajectory then interpolates between the classical action of the underlying field theory (at large $k$ ), and the full effective action (at $k=0$ ) [8, 9]. This method has been successfully applied to a range of problems, from condensed matter physics [11, 12] to particle physics [13, 14].

Here we study a system of fermions interacting through an attractive two-body contact potential. We take as our starting point an EFT that describes the $s$-wave scattering of two fermions with a $T$-matrix determined by the scattering length $a_{0}: T=-(4 \pi / M)\left(-1 / a_{0}-\right.$ $i p)^{-1}$. Here a positive scattering length corresponds to a system with a two-body bound 
state (and hence repulsive phase-shifts for low-energy scattering) and a negative scattering length to one without a bound state. The binding energy gets deeper as $a_{0}$ gets smaller, while the limit $a_{0} \rightarrow \pm \infty$ corresponds to a zero-energy bound state.

Since we are interested in the appearance of a gap in the fermion spectrum, we need to parametrise our effective action in a way that can describe the qualitative change in the physics when this occurs. A natural way to do this is to introduce a boson field whose vacuum expectation value (VEV) describes that gap and so acts as the corresponding order parameter 6]. This ERG approach is similar to that for the chiral phase transition in Refs. 13, 14]. At the start of the RG flow, the boson field is not dynamical and is introduced through a Hubbard-Stratonovich transformation of the four-point interaction. As we integrate out more and more of the fermion degrees of freedom by running $k$ to lower values, we generate dynamical terms in the bosonic effective action.

For simplicity we treat a single species of fermion, as in neutron matter. We introduce a boson field $\phi$ describing correlated pairs of fermions, which leads rather naturally to the following Ansatz for $\Gamma$ :

$$
\begin{aligned}
& \Gamma\left[\psi, \psi^{\dagger}, \phi, \phi^{\dagger}, \mu, k\right]=\int d^{4} x[ \phi^{\dagger}(x) \\
&\left.+\psi^{\dagger}\left(Z_{\phi} i \partial_{t}+\frac{Z_{m}}{2 m} \nabla^{2}\right) \phi(x)-U\left(\phi, \partial_{t}+\mu\right)+\frac{Z_{M}}{2 M} \nabla^{2}\right) \psi \\
&\left.-Z_{g}\left(\frac{i}{2} \psi^{\mathrm{T}} \sigma_{2} \psi \phi^{\dagger}-\frac{i}{2} \psi^{\dagger} \sigma_{2} \psi^{\dagger \mathrm{T}} \phi\right)\right],
\end{aligned}
$$

The field $\phi$ has a non-standard normalisation, since we shall relate its VEV to the gap $\Delta$ via $\Delta^{2}=\left\langle\phi^{\dagger} \phi\right\rangle$. This means that we have only a dimensionless coupling-constant renormalisation $Z_{g}$ for the boson-fermion coupling. The bosons carry twice the charge of a fermion, and so couple to the chemical potential via a term $2 \mu Z_{\phi} \phi^{\dagger} \phi$ which has been absorbed into the definition of the quadratic term in the potential $U$. (Here $M$ is the mass of the fermions in vacuum and $m$ is naively chosen to have the value $2 M$, but its real role is only to make $Z_{m}$ dimensionless.)

We expand the potential $U$ about its minimum to quartic order in the field [9, 12],

$$
U\left(\phi, \phi^{\dagger}\right)=u_{0}+u_{1}\left(\phi^{\dagger} \phi-\Delta^{2}\right)+\frac{1}{2} u_{2}\left(\phi^{\dagger} \phi-\Delta^{2}\right)^{2},
$$

where the $u_{n}$ are defined as the derivatives of $U$ at its minimum, which occurs where $\phi^{\dagger}=$ $\phi=\Delta$. The values of the $u_{n}$ run with the scale of the regulator, $k$. They also depend on the chemical potential $\mu$, the other external parameter in $\Gamma$. 
In the RG evolution, we start at high $k$ from a free bosonic action and gapless fermions. In this symmetric phase we have $u_{1}>0$ and $\Delta=0$, and so the VEV of $\phi$ is zero. When $k$ is lowered, we expect $u_{1}$ to decrease until it reaches zero. At this point there is a transition to a phase with spontaneously broken $U(1)$ symmetry and a fermion energy gap. Here the minimum moves away from $\Delta=0$ and, since we expand around the minimum of $U$, we take $u_{1}=0$ in Eq. (2). The bosonic excitations in this phase are gapless "Goldstone" bosons.

The other parameters in the action, namely the wave-function renormalisations $Z_{\phi, \psi}$, the kinetic mass renormalisations $Z_{m, M}$ and the coupling $Z_{g}$, are all evaluated in the background field corresponding to the minimum of the potential. These other parameters also run with $k$. For large values of $k$, the fermionic parameters $Z_{M}$ and $Z_{\psi}$ tend to unity, while the bosonic ones $Z_{\phi}$ and $Z_{m}$ tend to zero. We choose the normalisation of the field $\phi$ so that $Z_{g}$ tends to unity for large $k$. The chemical potential $\mu$ can be determined from $\partial \Gamma / \partial \mu=n$, where $n$ is the baryon-number density. To be able to study the whole range of phenomena from BCS to BEC (where $\mu$ becomes negative) we keep this density fixed, and allow $\mu$ to run during the RG evolution. Following Refs. 3, 5] we define a "Fermi momentum" $p_{F}$ in terms of density: $p_{F}=\left(3 \pi^{2} n\right)^{1 / 3}$. In the symmetric phase the fermion spectrum does not change and $p_{F}$ can be related to $\mu$ by $\mu=p_{F}^{2} /(2 M)$. However once a gap appears this connection is lost and indeed the whole idea of a Fermi surface may lose its meaning.

In the results presented here, we allow only $Z_{\phi}$ and the parameters in the potential to run independently since this is the minimal set needed to treat the bosons dynamically. We freeze the other coefficents $\left(Z_{\psi}, Z_{M}\right.$ and $\left.Z_{g}\right)$ to unity or, in the case of the boson kinetic mass, we have also explored setting $Z_{m}=Z_{\phi}$.

The evolution equation for $\Gamma$ in the ERG has a straightforward one-loop structure [9]. For constant $\mu$ it can be written

$$
\partial_{k} \Gamma=-\frac{i}{2} \operatorname{Tr}\left[\left(\boldsymbol{\Gamma}_{B B}^{(2)}-\mathbf{R}_{B}\right)^{-1} \partial_{k} \mathbf{R}_{B}\right]+\frac{i}{2} \operatorname{Tr}\left[\left(\boldsymbol{\Gamma}_{F F}^{(2)}-\mathbf{R}_{F}\right)^{-1} \partial_{k} \mathbf{R}_{F}\right] .
$$

Here $\Gamma_{F F(B B)}^{(2)}$ is the matrix containing second functional derivatives of the effective action with respect to the fermion (boson) fields and $\mathbf{R}_{B(F)}$ is a matrix containing the corresponding boson (fermion) regulators. A $2 \times 2$ matrix structure arises for the bosons because we treat $\phi$ and $\phi^{\dagger}$ as independent fields in order to include the number-violating condensate. A similar structure also appears for the fermions. By inserting our ansatz for $\Gamma$ into this equation we can turn it into a set of coupled equations for the various parameters discussed above. 
The one-loop structure of the ERG equations means that the evolution at large $k$ can be matched onto that of the underlying EFT [1], albeit for a non-standard choice of regularisation. In the bosonic sector, the regulator is an additional bilinear term in the fields. It has the matrix structure

$$
\mathbf{R}_{B}(q, k)=R_{B}(q, k) \operatorname{diag}(1,1),
$$

where $R_{B}(q, k)$ is a scalar function. This regulator provides an extra contribution to the single-particle energies, which should suppress the contributions of states with momenta $q \lesssim k$. As $k$ tends to zero $R_{B}(k)$ should vanish, so that we would recover the full effective action in the absence of any truncations. For large $k, R_{B}(q, k)$ should be large for momenta $q \lesssim k$, to give all these modes large energies. In particular, it should be of order $k^{2}$ if the behaviour of any integral for large $k$ is to reflect its order of divergence. For more discussion of the choice of regulator in this approach, see Ref. [15].

In the symmetric phase, our regulator for the fermions should be positive for particle states $\left(q>p_{F}\right)$ and negative for hole states $\left(q<p_{F}\right)$, so that it provides a $k$-dependent energy gap for excitations around the Fermi surface. It should have the structure

$$
\mathbf{R}_{F}\left(q, p_{F}, k\right)=\operatorname{sgn}(\epsilon(q)-\mu) R_{F}\left(q, p_{F}, k\right) \operatorname{diag}(1,-1),
$$

where $\epsilon(q)=q^{2} /(2 M)$. The function $R_{F}\left(q, p_{F}, k\right)$ should be peaked about $p_{F}$ to suppress the contributions of states with momenta $\left|q-p_{F}\right| \lesssim k$, but otherwise it should behave like the bosonic function. Note that the "Fermi surface" in the gapped phase no longer lies exactly at $p_{F}$, and there may not even be a well-defined Fermi surface. However, in that case a real gap has appeared in the spectrum and the regulator no longer plays a crucial role.

It is worth noting that we can recover the usual mean-field results if we include fermion loops only, ignoring all diagrams with virtual bosons. In this case the equation for the effective potential can be integrated analytically without making any truncation, and the results for $k=0$ coincide with those in Refs. 2, 3]. The initial conditions on the evolution are obtained by assuming that in vacuum our theory reproduces the scattering length $a_{0}$, and that the necessary subtraction are identical in matter and in vacuum. (See Ref. 1] and references therein for discussion of the vacuum problem.) The resulting running potential has the form

$$
U^{\mathrm{MF}}\left(\Delta^{2}, \mu, k\right)=\int \frac{d^{3} \boldsymbol{q}}{(2 \pi)^{3}}\left(\epsilon(q)-\mu+\frac{1}{2} \frac{\Delta^{2}}{\epsilon(q)}-\sqrt{E_{F R}(q, k)^{2}+\Delta^{2}}\right)+\frac{M}{4 \pi a_{0}} \Delta^{2},
$$


where we have introduced the short-hand notation

$$
E_{F R}(q, k)=\epsilon(q)-\mu+R_{F}\left(q, p_{F}, k\right) \operatorname{sgn}(\epsilon(q)-\mu) .
$$

For $k=0$ this has a closed-form expression in terms of an associated Legendre function, $P_{l}^{m}(y):$

$$
U^{\mathrm{MF}}\left(\Delta^{2}, \mu, 0\right)=\frac{k_{\Delta}{ }^{5}}{2 M \pi}\left(\frac{1}{8 a_{0} k_{\Delta}}-\frac{1}{15}\left(1+x_{0}{ }^{2}\right)^{\frac{3}{4}} P_{\frac{3}{2}}^{1}\left(-\frac{x_{0}}{\sqrt{1+x_{0}^{2}}}\right)\right) .
$$

where $k_{\Delta}=\sqrt{2 M \Delta}$ and $x_{0}=\mu / \Delta$. In the weakly attractive limit, $p_{F} a_{0} \rightarrow 0^{-}$, the chemical potential is directly related to the density by $\mu=\epsilon_{F}=p_{F}^{2} /(2 M)$, and minimising $U^{\mathrm{MF}}$ with respect to $\Delta$ leads to the standard result (see, e.g., Ref. [2])

$$
\Delta=\frac{8}{e^{2}} \epsilon_{F} \exp \left(-\frac{\pi}{2 p_{F}\left|a_{0}\right|}\right) \text {. }
$$

More generally, working at constant density, we can minimise $U$ subject to the subsidiary condition $\partial U / \partial \mu=n$. This gives the results from Ref. [3], which can be turned into a pair of equations for $\Delta$ and $\mu$,

$$
\begin{aligned}
& \Delta / \epsilon_{F}=\left(-3 \pi^{2}\left(x_{0}^{2}+1\right)^{1 / 4} P_{1 / 2}^{1}\left(y_{0}\right)\right)^{-2 / 3} \\
& \frac{1}{p_{F} a_{0}}=-\frac{2}{3} x_{0}\left(x_{0}^{2}+1\right)^{1 / 4}\left(P_{1 / 2}^{1}\left(y_{0}\right)-\frac{1}{y_{0}} P_{3 / 2}^{1}\left(y_{0}\right)\right)\left(\frac{\Delta}{\epsilon_{F}}\right)^{1 / 2}
\end{aligned}
$$

where $y_{0}=-x_{0}\left(x_{0}^{2}+1\right)^{-1 / 2}$. Note that, in this case,$\Delta / \epsilon_{F}$ can be eliminated from these results to leave a single equation for $x_{0}=\mu / \Delta$ in terms of $p_{F} a_{0}$.

We now turn to the full evolution equations. At the present level of truncation, all of these can be obtained from the evolution of the effective potential. It is convenient to write this as $U(\rho, \mu, k)$ where $\rho=\phi^{\dagger} \phi$. The density and $Z_{\phi}$ are then given by

$$
n=-\left.\frac{\partial U}{\partial \mu}\right|_{\rho=\Delta^{2}}, \quad Z_{\phi}=-\left.\frac{1}{2} \frac{\partial^{2} U}{\partial \rho \partial \mu}\right|_{\rho=\Delta^{2}} .
$$

By evaluating the loop integrals in Eq. (3) for a uniform boson field, we get evolution equation for $U$ at constant $\mu$,

$$
\begin{aligned}
\partial_{k} U= & -\frac{1}{Z_{\psi}} \int \frac{d^{3} \boldsymbol{q}}{(2 \pi)^{3}} \frac{E_{F R}}{\sqrt{E_{F R}^{2}+\rho}} \operatorname{sgn}(\epsilon(q)-\mu) \partial_{k} R_{F} \\
& +\frac{1}{2 Z_{\phi}} \int \frac{d^{3} \boldsymbol{q}}{(2 \pi)^{3}} \frac{E_{B R}}{\sqrt{E_{B R}^{2}-V_{B}^{2}}} \partial_{k} R_{B}
\end{aligned}
$$


where

$$
E_{B R}(q)=\frac{Z_{m}}{2 m} q^{2}+u_{1}+u_{2}\left(2 \rho-\Delta^{2}\right)+R_{B}(q, k), \quad V_{B}=u_{2} \rho,
$$

and $E_{F R}$ is defined above. Substituting our expansion on the left-hand side leads to a set of equations for the $u_{n}$, each of which is coupled to the coefficient of the next term through the running of $\Delta^{2}$, the position of the minimum of $U$. These equations have the form

$$
\partial_{k} u_{n}-u_{n+1} \partial_{k} \Delta^{2}=\left.\frac{\partial^{n}}{\partial \rho^{n}}\left(\partial_{k} U\right)\right|_{\rho=\Delta^{2}} .
$$

In the symmetric phase $\left(\Delta^{2}=0\right)$ they decouple. In the broken phase they do not and, for example, the left-hand-side of the equation for $\partial_{k} u_{2}$ contains the coefficent $u_{3}$. We could simply set $u_{3}=0$, but a better approximation can be obtained by substituting the form for $u_{3}(k)$ from the evolution with fermion loops only, as described above. This is the approach we adopt here for $u_{3}$ and similar coefficients in the equations for $n$ and $Z_{\phi}$. It has the advantage of providing an approximation that becomes exact in situations where boson loops can be neglected.

In order to follow the evolution at constant density we allow $\mu$ to run with $k$ and define the total derivative

$$
d_{k}=\partial_{k}+\left(d_{k} \mu\right) \frac{\partial}{\partial \mu},
$$

where $d_{k} \mu=d \mu / d k$. Applying this to $\partial U / \partial \mu$ and demanding that $n$ is constant $\left(d_{k} n=0\right)$ gives

$$
-2 Z_{\phi} d_{k} \Delta^{2}+\chi d_{k} \mu=-\left.\frac{\partial}{\partial \mu}\left(\partial_{k} U\right)\right|_{\rho=\Delta^{2}},
$$

where we have introduced the fermion-number susceptibility $\chi=\partial^{2} U /\left.\partial \mu^{2}\right|_{\rho=\Delta^{2}}$. The equations for the coefficients $u_{n}$ in the potential and $Z_{\phi}$ are

$$
\begin{aligned}
d_{k} u_{0}+n d_{k} \mu & =\left.\partial_{k} U\right|_{\rho=\Delta^{2}}, \\
d_{k} u_{1}-u_{2} d_{k} \Delta^{2}+2 Z_{\phi} d_{k} \mu & =\left.\frac{\partial}{\partial \rho}\left(\partial_{k} U\right)\right|_{\rho=\Delta^{2}}, \\
d_{k} u_{2}-u_{3} d_{k} \Delta^{2}+2 z_{\phi 1} d_{k} \mu & =\left.\frac{\partial^{2}}{\partial \rho^{2}}\left(\partial_{k} U\right)\right|_{\rho=\Delta^{2}}, \\
d_{k} Z_{\phi}-z_{\phi 1} d_{k} \Delta^{2}+\frac{1}{2} \chi^{\prime} d_{k} \mu & =-\left.\frac{1}{2} \frac{\partial^{2}}{\partial \mu \partial \rho}\left(\partial_{k} U\right)\right|_{\rho=\Delta^{2}},
\end{aligned}
$$

where we have defined

$$
z_{\phi 1}=-\left.\frac{1}{2} \frac{\partial^{3} U}{\partial^{2} \rho \partial \mu}\right|_{\rho=\Delta^{2}} \quad \chi^{\prime}=\left.\frac{\partial^{3} U}{\partial \mu^{2} \partial \rho}\right|_{\rho=\Delta^{2}} .
$$


The driving terms in these evolution equations are given by appropriate derivatives of Eq. (12). In the symmetric phase we evaluate these expressions at $\Delta^{2}=0$. The driving term of Eq. (16) vanishes in this case, and hence $\mu$ remains constant. In the broken phase we keep $\Delta^{2}$ non-zero and set $u_{1}=0$.

On the left-hand sides of these equations, the coefficients $u_{3}, z_{\phi 1}, \chi$ and $\chi^{\prime}$ all correspond to terms outside our present truncation and so we replace them by their expressions from evolution with fermion loops only. This approximation provides a closed set of equations, whose solutions agree with the exact results when boson loops are neglected. Underlying it is an assumption is that bosonic contributions to the evolution are not too large compared to fermionic ones. We shall investigate this assumption below, and find that it seems to hold for a wide range of values of $p_{F} a_{0}$. In future work, we hope to be able to check it further by including more bosonic terms in our ansatz for $\Gamma$ and examining convergence with respect to the number of terms.

We integrate the resulting differential equations numerically. In this work, we use regulator functions with the forms

$$
R_{F}\left(q, k ; p_{F}, \sigma\right)=\frac{k^{2}}{2 M} \theta\left(q-p_{F}, k ; \sigma\right), \quad R_{B}(q, k ; \sigma)=\frac{k^{2}}{2 m} \theta(q, k ; \sigma) .
$$

where $\theta$ is the smoothed step-function

$$
\theta(q, k ; \sigma)=\frac{1}{2 \operatorname{erf}(1 / \sigma)}\left[\operatorname{erf}\left(-\frac{(q+k)}{k \sigma}\right)+\operatorname{erf}\left(-\frac{(q-k)}{k \sigma}\right)\right],
$$

with $\sigma$ being a parameter controlling the sharpness of the step. The derivative $\partial_{k} \theta$ falls off for $k \rightarrow \infty$ and so $\partial_{k} R_{B, F}$ provides a UV cut-off on the loop integrals in Eq. (12) [9].

We run the evolution down towards $k=0$ starting from some large, fixed scale, $k=K$. The initial conditions for this are obtained by matching onto the standard evolution in vacuum for $k \geq K$ using Eq. (6) and related expressions. Differentiating $U^{\mathrm{MF}}$ with respect to $\Delta^{2}$ at $\Delta=0$ we get the initial condition on $u_{1}$,

$$
u_{1}(K)=-\frac{M}{4 \pi a_{0}}+\frac{1}{2} \int \frac{d^{3} \boldsymbol{q}}{(2 \pi)^{3}}\left(\frac{\operatorname{sgn}(\epsilon(q)-\mu)}{E_{F R}(q, K)}-\frac{1}{\epsilon(q)}\right) .
$$

The second term in the integral contains exactly the same linearly divergent term as in the free inverse $T$ matrix. It cancels with the similar divergence in the first term to leave a finite result. This result is linear in $K$, reflecting the divergence of the underlying integral. It also contains a correction for the fact that our regulator depends on the density (through $p_{F}$ ). The initial values of $u_{2}$ and $Z_{\phi}$ can be obtained similarly from second derivatives of $U^{\mathrm{MF}}$. 

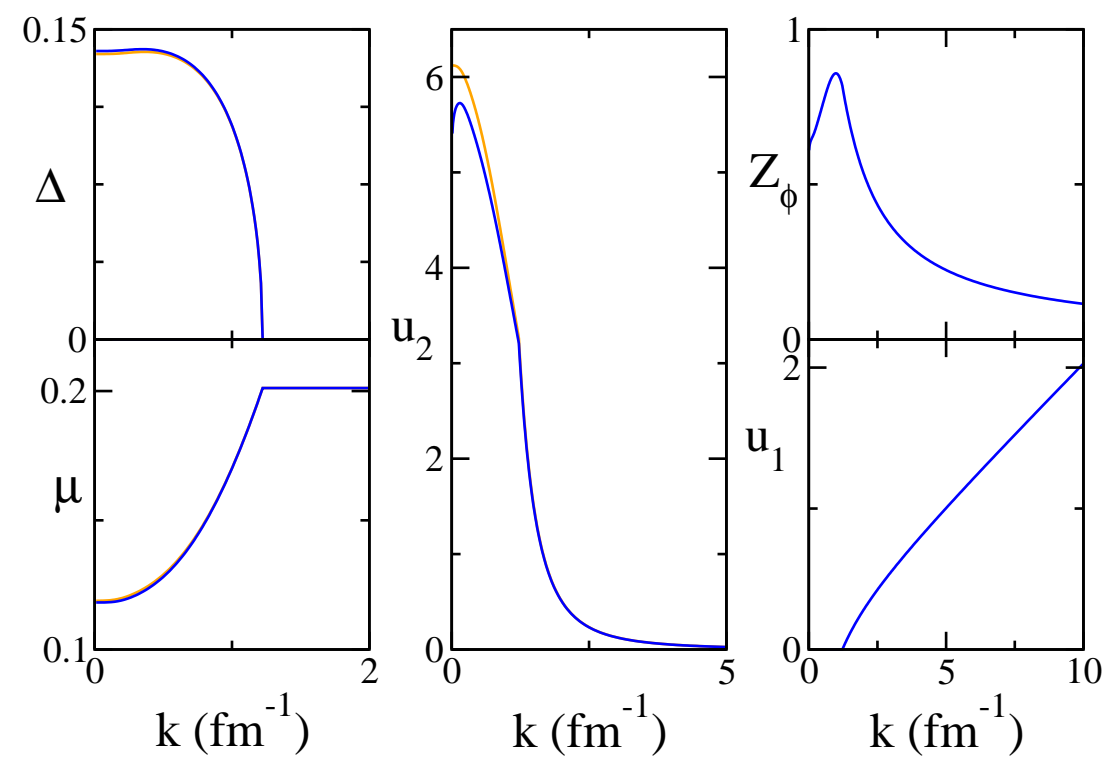

FIG. 1: Numerical solutions to the evolution equations for infinite $a_{0}$ and $p_{F}=1.37 \mathrm{fm}$, starting from $K=16 \mathrm{fm}^{-1}$. We show the evolution of all relevant parameters for the cases of fermion loops only (orange/grey lines), and of bosonic loops with a running $Z_{\phi}$ (blue/black lines). All quantities are expressed in appropriate powers of $\mathrm{fm}^{-1}$.

Although our results can be applied to many systems, for definiteness we concentrate initially on parameter values relevant to applications to neutron matter: $M=4.76 \mathrm{fm}^{-1}$, $p_{F}=1.37 \mathrm{fm}^{-1}$, and large two-body scattering lengths $\left(\left|a_{0}\right| \gtrsim 1 \mathrm{fm}\right)$. We then explore a wider range of values for $p_{F} a_{0}$. We have checked for dependence of our results on the starting scale $K$ and find that this is undetectable as long as $K>5 \mathrm{fm}^{-1}$ (about $4 p_{F}$ ). Similarly, we get numerically indistinguishable results for a range of values of the width parameter $\sigma$.

Some typical solutions for the evolution equations are given in Fig. 11 for the case of infinite $a_{0}$. We compare two different approximation schemes, one where we have fermion loops only, and one where we include boson loops as well and we allow $Z_{\phi}$ to run. For large values of $k$ the system remains in the symmetric phase. At $k_{\text {crit }} \simeq 1.2 \mathrm{fm}^{-1}, u_{1}$ vanishes and below that point the system is in the broken phase where we plot $\Delta$ instead of $u_{1}$.

One immediate observation is that the contributions of boson loops are small. Indeed in the symmetric phase their inclusion has essentially no effect. Below the transition they do become visible, particularly in $u_{2}$. However their effects on the gap are even smaller, at most $\sim 1 \%$ for $1 /\left|p_{F} a_{0}\right|<1$. In this region, the main effect of the bosons is a small enhancement 


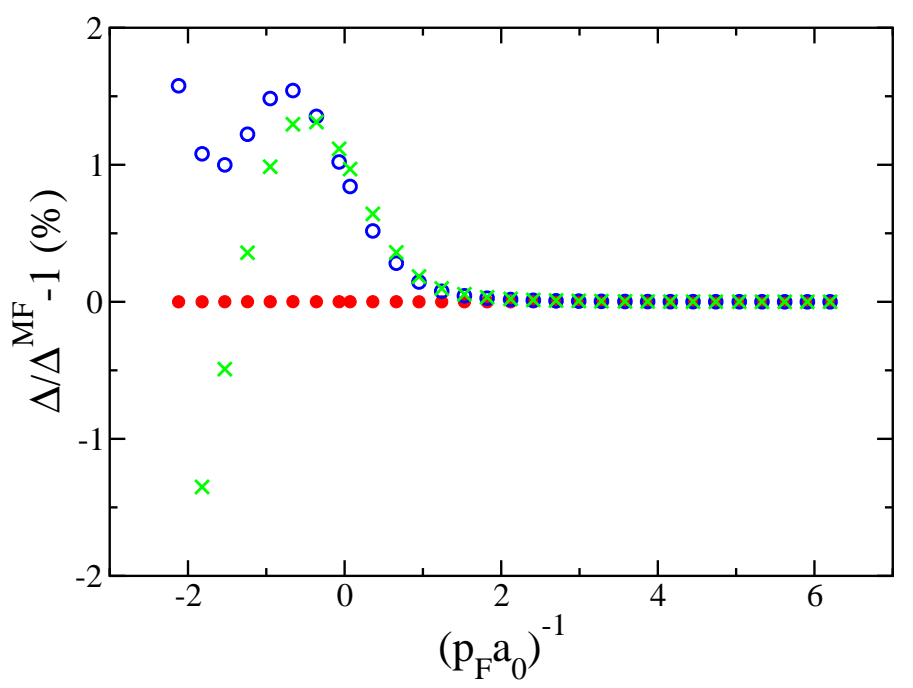

FIG. 2: Fractional deviations (in \%) of the gaps for various numerical solutions from the analytic mean-field result. All cases are for $p_{F}=1.37 \mathrm{fm}^{-1}$. Solid dots (red) denote the results from a calculation with fermion loops only, the open circles (blue) show the results when boson loops are added, and the crosses (green) show the effect of allowing $Z_{\phi}$ to run as well.

of the gap related to the reduction in $u_{2}$ as $k \rightarrow 0$. Not plotted are results including boson loops but with $Z_{\phi}$ fixed at unity; the results are vitually indistinguishable from those with running $Z_{\phi}$. The same is true if we set $Z_{m}=Z_{\phi}$ rather than $Z_{m}=1$.

It is also instructive to examine the behaviour of the gap over a wider range of $1 /\left(p_{F} a_{0}\right)$. The overall picture the same as found in Ref. [5] for fermion loops only, with a crossover from BCS pairing (with positive $\left.\mu \simeq \epsilon_{F}\right)$ for $1 /\left(p_{F} a_{0}\right)<0$ to BEC (with increasingly negative values for $\mu$ ) for $1 /\left(p_{F} a_{0}\right)>0$. For negative $1 /\left(p_{F} a_{0}\right)$, the gap lies close to the exponential curve of the weak coupling limit, Eq. (9). However a closer look at these results, as in Fig. 2, shows that deviations from mean field behaviour are present in this region and become increasingly noticable for weaker couplings or lower densities.

We are not able to follow our results beyond $1 /\left(p_{F} a_{0}\right) \sim-2$. This is because of the nonanalyticity of the effective action for small gaps which means that our expansion Eq. (2) is no longer adequate. For example, the fermion loops contain a term $\phi^{\dagger} \phi \log \left(\phi^{\dagger} \phi\right)$ which gives a divergent contribution to $u_{2}$. Although the complete fermionic part has been incorporated in our equations, the analogous boson effects are not included. Some of our results suggest that the gap vanishes as a power rather than an exponential, but a definite conclusion will 
have to await a more complete calculation.

The dominance of fermion loops for most values of $1 /\left(p_{F} a_{0}\right)$ may seem surprising. However this is the region of BEC or the crossover to it, where the mean boson field (the gap) is large. In fact the gap is particularly insensitive to boson fluctuations as a result of cancellations between the direct contribution to the running of $\Delta^{2}$ and indirect ones via $u_{2}$. Contributions to other parameters such as $u_{2}$ and $Z_{\phi}$ are larger, $\sim 10 \%$ for $1 /\left(p_{F} a_{0}\right) \simeq 0$. Our results imply that these effects are likely to be important for neutron matter, where calculations with realistic interactions lead to gaps of at most $5 \mathrm{MeV}\left(\sim 0.1 \epsilon_{F}\right)[16]$.

However, if we were to apply our current calculation to neutron matter, we would find a gap comparable to $\epsilon_{F}$, of the order of $30 \mathrm{MeV}$. Fayans has given a simple explanation for the smaller values found using more sophisticated treatments [17]. ${ }^{1}$ The argument can be given most succinctly for weak coupling, where the gap satisfies a generalisation of Eq. (9),

$$
\Delta=\left(8 / e^{2}\right) \epsilon_{F} \exp \left(-(\pi / 2) \cot \left(\delta\left(p_{F}\right)\right)\right)
$$

For nucleon-nucleon scattering, $\cot \delta$ increases relatively quickly with momentum and the resulting reduction in the gap is substantial. We therefore expect that an extension of our approach to include the effective range should capture this physics.

There a number of improvements which could be made to our approach. Adding an effective range is clearly an important one. Another is the evolution of the boson kinetic mass $\left(Z_{m}\right)$ since this will allow a scaling analysis of boson loops for small gaps. We should also include running of the fermion renormalisation constants and "Yukawa" coupling, to provide a full treatment of the action (11). Beyond that we would like to treat explicitly the particle-hole channels (RPA phonons) since these contain important physics. They will also allow us to remove the "Fierz ambiguity" associated with our bosonisation of the underlying contact interaction 21]. We plan to explore these extensions in future studies.

This research was funded by the EPSRC.

[1] P. F. Bedaque and U. van Kolck, Ann. Rev. Nucl. Part. Sci. 52 (2002) 339 nucl-th/0203055.

\footnotetext{
${ }^{1}$ A similar result can be obtained using the results of Ref. [18] or [19]. Note that the derivation of the effective range formula in the second reference is unfortunately incorrect, due to the use of a misleading result quoted in Ref. 20].
} 
[2] T. Papenbrock and G. Bertsch, Phys. Rev. C59 (1999) 2052 nucl-th/9811077.

[3] M. Marani, F. Pistolesi and G. C. Strinati, Eur. Phys. J. B 1 (1998) 151 cond-mat/9703160.

[4] D. M. Eagles, Phys. Rev. 186 (1969) 456; A. J. Leggett, in Modern Trends in the Theory of Condensed Matter, eds. A. Pekalski and J. Przystawa, Lect. Notes in Pys. 115 (Springer Verlag, Berlin, 1980), p. 13.; P. Nozieres and S. Schmitt-Rink, J. Low. Temp. Phys. 59 (1985), 159.

[5] E. Babaev, Phys. Rev. B 63 (2001) 184514 cond-mat/0010085.

[6] S. Weinberg, Nucl. Phys. B413 (1994) 567 cond-mat/9306055.

[7] K. G. Wilson and J. G. Kogut, Phys. Rep. 12C (1974) 75.

[8] C. Wetterich, Phys. Lett. B301 (1993) 90.

[9] J. Berges, N. Tetradis and C. Wetterich, Phys. Rept. 363 (2002) 223 hep-ph/0005122.

[10] S. Weinberg, The quantum theory of fields, Vol. 2, Chapter 16 (Cambridge University Press, 1996).

[11] N. Tetradis and C. Wetterich, Nucl. Phys. B422 (1994) 541 hep-ph/9308214.

[12] B. Delamotte, D. Mouhanna and M. Tissier, Phys. Rev. B 69 (2004) 134413 cond-mat/0309101.

[13] D.-U. Jungnickel and C. Wetterich, Phys. Rev. D 53 (1996) 5142 hep-ph/9505267.

[14] J. Berges, D.-U. Jungnickel and C. Wetterich, Eur. Phys. J. C13 (2000) 323 hep-ph/9811347.

[15] D. Litim, JHEP 0111 (2001) 059 hep-th/0111159.

[16] D. J. Dean and M. Hjorth-Jensen, Rev. Mod. Phys. 75 (2003) 607 nucl-th/0210033

[17] S. A. Fayans and D. Zawischa, Int. J. Mod. Phys. B15 (2001) 1684 nucl-th/0009034.

[18] V. A. Khodel, V. V. Khodel, and J. W. Clark, Nucl. Phys. A598 (1996) 390.

[19] Ø. Elgarøy and M. Hjorth-Jensen, Phys. Rev. C57 (1998) 1174 nucl-th/9708026.

[20] K. Chadan and P. C. Sabatier, Inverse problems in scattering theory, 2nd ed. (Springer Verlag, New York, 1989)

[21] J. Jaeckel and C. Wetterich, Phys. Rev. D 68 (2003) 025020 hep-ph/0207094. 